\title{
Receiver Multiuser Diversity Aided Multi-Stage Minimum Mean-Square Error Detection for Heavily Loaded DS-CDMA and SDMA Systems
}

\author{
Lie-Liang Yang, Senior Member, IEEE
}

\begin{abstract}
By investigating the conditions required for successive interference cancellation multiuser detectors (SIC-MUD) to achieve near-optimum BER performance, we propose and investigate a so-called receiver multiuser diversity aided multi-stage minimum mean-square error MUD (RMD/MS-MMSE MUD), which is operated in the SIC principles. The BER performance of the RMD/MS-MMSE MUD is investigated in association with both the direct-sequence code-division multiple-access (DSCDMA) over either Gaussian or Rayleigh fading channels, and the space-division multiple-access (SDMA) over Rayleigh fading channels. Furthermore, we consider both full-load and overload scenarios in comparison with the spreading factor $N$ of DSCDMA and the number of receive antennas $N$ in SDMA. Our studies show that the RMD/MS-MMSE MUD is highly efficient for both full-load and overload systems. Specifically, the RMD/MS-MMSE MUD for the full-load systems of moderate size is capable of attaining the BER performance similar to that of the optimum maximum likelihood MUD (ML-MUD). For the overload systems, it can allow a DS-CDMA or SDMA system to support $K=2 N$ users and still achieve much better BER performance than a corresponding DS-CDMA or SDMA system using conventional MMSE-MUD to support $K=N$ users.
\end{abstract}

Index Terms-MIMO, DS-CDMA, SDMA, multiuser detection, maximum likelihood, minimum mean-square error, interference cancellation, reliability, ordered statistics.

\section{INTRODUCTION}

I $\mathrm{N}$ multiuser detection, the optimum maximum a-posteriori (MAP) and maximum likelihood (ML) multiuser detectors (MUDs) [1]-[3] are capable of achieving the optimum BER performance that is close to the single-user BER performance bound. However, the complexity of these optimum MUDs is exponentially proportional to the number of users detected, which becomes extreme even when a moderate number of users are involved. Consequently, the practical application of the optimum MUDs is limited. For this sake, since the invention of MUD [1], great effort has been made for design of low-complexity high-efficiency suboptimum MUD algorithms and numerous MUD algorithms have been proposed, as evidenced by [3]-[9] and the references therein. However, the suboptimum algorithms designed so far are either still too complex to be implemented in practice or achieve much worse BER performance than the optimum MUDs [3]-[5].

In this paper, we first introduce a novel concept of receiver multiuser diversity (RMD), in order to study the sparsity of

Paper approved by N. Al-Dhahir, the Editor for Space-Time, OFDM and Equalization of the IEEE Communications Society. Manuscript received May 14, 2009; revised January 15, 2010 and April 26, 2010.

This work was presented in part at IEEE VTC 2010 Spring, Taipei, Taiwan.

The author is with the School of Electronics and Computer Science, University of Southampton, SO17 1BJ, UK (e-mail: 1ly@ecs.soton.ac.uk).

Digital Object Identifier 10.1109/TCOMM.2010.091710.090263 reliabilities, which is critical for design of high-efficiency SICMUDs. Our studies show that, when employing the MAP principles for detection, RMD exists in both Gaussian and Rayleigh fading channels. Therefore, we propose and investigate a so-called RMD/MS-MMSE MUD, which belongs to the family of SIC-MUDs and is operated in the principles similar to that of the V-BLAST systems [7]-[9]. We motivate to reveal the fact that a low-complexity SIC-MUD is capable of achieving the BER performance very close to that of the optimum MUDs, provided that the following two conditions are satisfied; Firstly, the reliabilities of different users can be measured near independently in an optimum way. Secondly, the reliabilities are highly sparse, resulting in that some users can be detected much more reliably than some other users. This paper demonstrates that the above-mentioned two conditions can be closely met by the multiuser systems using the conventional minimum mean-square error MUD (MMSEMUD), when the reliabilities are measured approximately in MAP sense [10]. Hence, in our RMD/MS-MMSE MUD, an approximate MAP-based reliability measurement (RM) scheme is then proposed for ordering the users to be detected. In this paper, the performance of the RMD/MS-MMSE MUD is investigated in the context of both DS-CDMA and SDMA systems, when they are either full-load or overload. Our studies show that the BER performance achieved by the RMD/MS-MMSE MUD for full-load systems is very close to that of the optimum MUD. The RMD/MS-MMSE MUD is high-efficiency, even when a DS-CDMA or SDMA system is overload.

Note that the complexity of the RMD/MS-MMSE MUD is similar as that of a conventional MMSE-SIC scheme [3], [4], [6] or of a V-BLAST scheme [7]-[9]. It can be implemented using the low-complexity approaches proposed, e.g., in [12][14].

The rest of the paper is organized as follows. In Section II, we analyze and illustrate the RMD. Section III gives an essential overview of system model and MMSE-MUD. In Section IV, RMD/MS-MMSE MUD is described and analyzed, while in Section V a range of BER performance results are provided. Finally, in Section VI, we summarize our conclusions.

\section{Principles of Receiver Multiuser Diversity}

Let us consider detecting simultaneously $K$ binary phaseshift keying (BPSK) signals based on the decision variables

$$
y_{k}=b_{k}+n_{k}, k=1,2, \ldots, K
$$


where $b_{k} \in\{+1,-1\}$ is assumed to be an independent identically distributed (iid) random variable with $P\left(b_{k}=\right.$ $+1)=P\left(b_{k}=-1\right)=0.5$ and $n_{k}$ obeys Gaussian distribution with zero mean and a variance of $\sigma^{2}=1 /\left(2 \gamma_{k}\right)$, where $\gamma_{k}=\alpha_{k}^{2} \gamma_{b}$ denotes the instantaneous signal-to-noise ratio (SNR) per bit, while $\gamma_{b}$ denotes the average SNR per bit. Furthermore, $\alpha_{k}=1$ corresponds to the Gaussian channel scenarios, while $\alpha_{k}$ obeys the iid Rayleigh distribution associated with $E\left[\alpha_{k}^{2}\right]=1$ when communicating over Rayleigh fading channels.

In this section, we investigate the reliabilities for detection of $\left\{b_{k}\right\}$ and the BER performance of the $K$ bits on the $K$ reliability levels without minding which bit is received from which user. The studies show that the BER performance corresponding to the $K$ reliability levels is highly diverse in both Gaussian and Rayleigh fading channels. Hence, we refer to this type of diversity as the receiver multiuser diversity (RMD), since the diversity is due to the multiple users involved. Furthermore, 'receiver' is explicitly used to emphasize that the RMD does not invoke any assistance from the transmitters.

The reliability of detecting $b_{k}$ can be optimally measured by the magnitude of the posteriori log-likelihood ratio (LLR) [15]

$$
L_{k}=\left|\ln \left[\frac{f\left(b_{k}=+1 \mid y_{k}\right)}{f\left(b_{k}=-1 \mid y_{k}\right)}\right]\right|=\left|\ln \left[\frac{f\left(y_{k} \mid b_{k}=+1\right)}{f\left(y_{k} \mid b_{k}=-1\right)}\right]\right|
$$

Applying the Gaussian probability density function (PDF) of $y_{k}$ into (2) yields

$$
L_{k}=\left|4 \gamma_{k} y_{k}\right| \triangleq \gamma_{k}\left|y_{k}\right|, k=1,2, \ldots, K
$$

Given $\gamma_{k}$ and $b_{k}$, it can be readily shown that $q_{k}=\gamma_{k} y_{k}$ follows the PDF

$$
\begin{aligned}
f_{q_{k}}\left(y \mid \gamma_{k}, b_{k}\right)= & \frac{1}{\sqrt{\pi \gamma_{k}}} \exp \left(-\frac{\left(y-\gamma_{k} b_{k}\right)^{2}}{\gamma_{k}}\right), \\
& k=1,2, \ldots, K
\end{aligned}
$$

and, furthermore, the PDF of $L_{k}, k=1, \ldots, K$, of (3) can be derived as

$$
\begin{gathered}
f_{L_{k}}\left(y \mid \gamma_{k}\right)=f_{q_{k}}\left(y \mid \gamma_{k}, b_{k}\right)+f_{q_{k}}\left(-y \mid \gamma_{k}, b_{k}\right) \\
=\frac{1}{\sqrt{\pi \gamma_{k}}}\left[\exp \left(-\frac{\left(y+\gamma_{k}\right)^{2}}{\gamma_{k}}\right)+\exp \left(-\frac{\left(y-\gamma_{k}\right)^{2}}{\gamma_{k}}\right)\right], \\
y \geq 0,
\end{gathered}
$$

Notice here that $f_{L_{k}}\left(y \mid \gamma_{k}\right)$ is independent of $b_{k}$, due to the symmetric property of $f_{q_{k}}\left(y \mid \gamma_{k},-1\right)=f_{q_{k}}\left(-y \mid \gamma_{k},+1\right)$ and $f_{q_{k}}\left(y \mid \gamma_{k},+1\right)=f_{q_{k}}\left(-y \mid \gamma_{k},-1\right)$. Furthermore, we note that (5) represents the normalized PDF of $L_{k}=\left|\gamma_{k} y_{k}\right|$. The PDF of $L_{k}=\left|4 \gamma_{k} y_{k}\right|$ can be readily obtained from (5) with replacing $y$ by $y / 4$ and multiplying the result by a factor of $1 / 4$.

For communications over Gaussian channels corresponding to $\alpha_{k}=1$, (4) and (5) are the desired PDFs for $q_{k}$ and $L_{k}$ with $\gamma_{k}=\gamma_{b}$, which are hence represented correspondingly by $f_{q_{k}}\left(y \mid b_{k}\right)$ and $f_{L_{k}}(y)$. When considering communications over Rayleigh fading channels, the condition of $\gamma_{k}$ on $q_{k}$ and $L_{k}$ need to be averaged out. Using the fact that the $K$ user signals are independent, the unconditional PDF of $q_{k}$ can be obtained as

$$
f_{q_{k}}\left(y \mid b_{k}\right)=\int_{0}^{\infty} f_{q_{k}}\left(y \mid \gamma_{k}, b_{k}\right) f\left(\gamma_{k}\right) d \gamma_{k}
$$

where $\gamma_{k}$ is exponentially distributed with the PDF of $f\left(\gamma_{k}\right)=$ $\gamma_{b}^{-1} e^{-\gamma_{k} / \gamma_{b}}$. Upon substituting this PDF and (4) into (6) and after some arrangements, we obtain

$f_{q_{k}}\left(y \mid b_{k}\right)=\frac{e^{2 b_{k} y}}{\sqrt{\pi} \gamma_{b}} \int_{0}^{\infty} \gamma_{k}^{-1 / 2} \exp \left(-\left[\frac{y^{2}}{\gamma_{k}}+\left(1+\frac{1}{\gamma_{b}}\right) \gamma_{k}\right]\right) d \gamma_{k}$

Using (3.471.9) of [16], we arrive at

$$
\begin{gathered}
f_{q_{k}}\left(y \mid b_{k}\right)=\frac{2 e^{2 b_{k} y}}{\sqrt{\pi} \gamma_{b}}\left(\sqrt{\frac{y^{2} \gamma_{b}}{1+\gamma_{b}}}\right)^{1 / 2} K_{1 / 2}\left(2 \sqrt{\frac{y^{2}\left(1+\gamma_{b}\right)}{\gamma_{b}}}\right), \\
k=1,2, \ldots, K
\end{gathered}
$$

where $K_{v}(x)$ denotes the modified Bessel function and, according to (8.469.3) of [16], $K_{1 / 2}(x)=\sqrt{\pi / 2 x} e^{-x}$. Using this result into (8), finally, we obtain the unconditional PDF of $q_{k}$ in Rayleigh fading channels, which is

$$
\begin{gathered}
f_{q_{k}}\left(y \mid b_{k}\right)=\sqrt{\frac{1}{\gamma_{b}\left(1+\gamma_{b}\right)}} \exp \left(-2 \sqrt{\frac{1+\gamma_{b}}{\gamma_{b}}}|y|+2 b_{k} y\right), \\
k=1,2, \ldots, K
\end{gathered}
$$

Remembering that $L_{k}=\left|q_{k}\right|$, then, similar to (5), the PDF of $L_{k}$ in Rayleigh fading channels can be obtained as

$$
\begin{gathered}
f_{L_{k}}\left(y \mid b_{k}\right)=f_{q_{k}}\left(y \mid b_{k}\right)+f_{q_{k}}\left(-y \mid b_{k}\right) \\
=\sqrt{\frac{1}{\gamma_{b}\left(1+\gamma_{b}\right)}\left[e^{2 y}+e^{-2 y}\right] \exp \left(-2 \sqrt{\frac{1+\gamma_{b}}{\gamma_{b}}} y\right),} \\
y \geq 0,
\end{gathered}
$$

which is also independent of $b_{k}$.

Let $F_{q_{k}}\left(y \mid b_{k}\right)$ and $F_{L_{k}}(y)$ denote the cumulative distribution functions (CDFs) of $q_{k}$ and $L_{k}$. Then, in the context of Gaussian channels, we have

$$
\begin{aligned}
& F_{q_{k}}\left(y \mid b_{k}\right)=Q\left(-\sqrt{\frac{2}{\gamma_{b}}} y+\sqrt{2 \gamma_{b}} b_{k}\right) \\
& F_{L_{k}}(y)=1-Q\left(\sqrt{\frac{2}{\gamma_{b}}} y+\sqrt{2 \gamma_{b}}\right)-Q\left(\sqrt{\frac{2}{\gamma_{b}}} y-\sqrt{2 \gamma_{b}}\right)
\end{aligned}
$$

where $Q(x)$ is the Gaussian $Q$-function defined as $Q(x)=(2 \pi)^{-1 / 2} \int_{x}^{\infty} e^{-t^{2} / 2} d t$. By contrast, when considering Rayleigh fading channels, the CDFs of $F_{q_{k}}\left(y \mid b_{k}\right)$ and $F_{L_{k}}(y)$ can be expressed as

$$
F_{q_{k}}\left(y \mid b_{k}\right)= \begin{cases}\frac{\exp \left(2\left[\sqrt{\frac{1+\gamma_{b}}{\gamma_{b}}}+b_{k}\right] y\right)}{2\left(1+\gamma_{b}+b_{k} \sqrt{\gamma_{b}\left(1+\gamma_{b}\right)}\right)}, & y \leq 0 \\ 1-\left(\frac{1}{2}+\frac{b_{k}}{2} \sqrt{\frac{\gamma_{b}}{1+\gamma_{b}}}\right) & \\ \quad \times \exp \left(-2\left[\sqrt{\frac{1+\gamma_{b}}{\gamma_{b}}}-b_{k}\right] y\right), & y \geq 0\end{cases}
$$

$$
\begin{gathered}
F_{L_{k}}(y)=1-\frac{1}{2}\left(1-\sqrt{\frac{\gamma_{b}}{1+\gamma_{b}}}\right) \exp \left(-2\left[1+\sqrt{\frac{1+\gamma_{b}}{\gamma_{b}}}\right] y\right) \\
-\frac{1}{2}\left(1+\sqrt{\frac{\gamma_{b}}{1+\gamma_{b}}}\right) \exp \left(2\left[1-\sqrt{\frac{1+\gamma_{b}}{\gamma_{b}}}\right] y\right)
\end{gathered}
$$




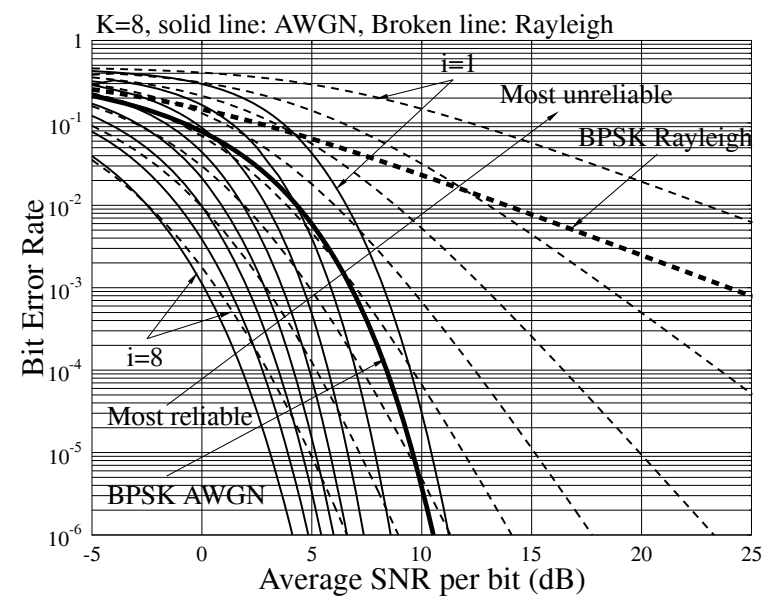

Fig. 1. BER versus average SNR per bit $\left(\gamma_{b}\right)$ for the bits at different reliability levels, where $i=1$ and 8 correspond to the most unreliable and most reliable bits, respectively.

Let us now order in ascent the reliabilities as

$$
L_{(1)} \leq L_{(2)} \leq \cdots \leq L_{(K)}
$$

where $L_{(i)}, i=1, \ldots, K$, represents the reliability of the $i$ th most unreliable in $\left\{L_{1}, L_{2}, \ldots, L_{K}\right\}, L_{(1)}$ and $L_{(K)}$ correspond to the most unreliable bit and the most reliable bit, respectively. Then, according to the derivation in the appendix, the BER of the bits at the $i$ th reliability level for communications over Gaussian channels can be expressed as

$$
\begin{array}{rl}
P_{E}(i)= & K\left(\begin{array}{c}
K-1 \\
i-1
\end{array}\right) \frac{1}{\sqrt{2 \pi}} \int_{\sqrt{2 \gamma_{b}}}^{\infty} \exp \left(-\frac{t^{2}}{2}\right) \\
& \times\left[1-Q(t)-Q\left(t-2 \sqrt{2 \gamma_{b}}\right)\right]^{i-1} \\
& \times\left[Q(t)+Q\left(t-2 \sqrt{2 \gamma_{b}}\right)\right]^{K-i} d t \\
i & i=1,2, \ldots, K
\end{array}
$$

By contrast, when communicating over Rayleigh fading channels, the BER of the bits at the $i$ th reliability level can be formulated as

$$
\begin{aligned}
P_{E}(i)= & \sum_{m=0}^{i-1} \sum_{n=0}^{K-i+m}(-1)^{m} K\left(\begin{array}{c}
K-1 \\
i-1
\end{array}\right)\left(\begin{array}{c}
i-1 \\
m
\end{array}\right)\left(\begin{array}{c}
K-i+m \\
n
\end{array}\right) \\
& \times \frac{D A^{n} B^{K-i+m-n}}{n+1-(K-i+m-n) C}, i=1,2, \ldots, K
\end{aligned}
$$

where, by definition,

$$
\begin{aligned}
A & =\frac{1}{2}\left(1-\sqrt{\frac{\gamma_{b}}{1+\gamma_{b}}}\right), \quad B=\frac{1}{2}\left(1+\sqrt{\frac{\gamma_{b}}{1+\gamma_{b}}}\right) \\
C & =2 \sqrt{\gamma_{b}\left(1+\gamma_{b}\right)}-2 \gamma_{b}-1 \\
D & =\frac{1}{2\left(\sqrt{\gamma_{b}\left(1+\gamma_{b}\right)}+\gamma_{b}+1\right)}
\end{aligned}
$$

Fig. 1 illustrates the BER evaluated by (16) and (17) for the Gaussian and Rayleigh fading channels, respectively, for the case of $K=8$. In the figure, the average (or unordered) BER of the BPSK scheme is also depicted. Explicitly, we can observe that the BER at different reliability levels is highly diverse, when communicating over both Gaussian and Rayleigh fading channels. This observation implies that RMD exists in both Gaussian and fading channels, in contrast to the other type of diversity, such as frequency-diversity, spatial-diversity, or the multiuser diversity so far considered in literature, which only exists in fading environments. Furthermore, the average BER is generally dominated by the several, typically two or three, most unreliable bits.

Having discussed the principles of the RMD, let us below consider the RMD/MS-MMSE MUD for DS-CDMA or SDMA systems.

\section{OVERVIEW OF SYSTEM MODEL AND MMSE DETECTION}

The MIMO equation for both DS-CDMA and SDMA systems supporting $K$ users can be expressed as

$$
y=H b+n
$$

where $\boldsymbol{y}$ and $\boldsymbol{n}$ are $N$-length complex-valued observation vector and noise vector, $\boldsymbol{b}=\left[b_{1}, b_{2}, \cdots, b_{K}\right]^{T}$ contains the data symbols transmitted by the $K$ users, $b_{k} \in\{+1,-1\}$, and $\boldsymbol{H}=\left[\boldsymbol{h}_{1}, \boldsymbol{h}_{2}, \cdots, \boldsymbol{h}_{K}\right]$ is an $(N \times K)$ matrix. For DSCDMA systems, the matrix $\boldsymbol{H}$ can be written as $\boldsymbol{H}=\boldsymbol{C A}$, where $C$ is an $(N \times K)$ spreading matrix with the $k$ th column representing the $k$ th user's DS spreading sequence. The matrix $A=\operatorname{diag}\left\{a_{1}, a_{2}, \cdots, a_{K}\right\}$ is an $(K \times K)$ diagonal matrix, where for AWGN channels $a_{k}=e^{j \theta_{k}}$ and for Rayleigh fading channels $a_{k}$ obeys iid complex Gaussian distribution with zero mean and unity variance. By contrast, for SDMA systems, each element of $H$ is assumed an iid complex Gaussian random variable with zero mean and a variance of $1 / N$. For both DS-CDMA and SDMA, we assume that the matrix $\boldsymbol{H}$ is normalized to satisfy $E\left[\boldsymbol{H}^{H} \boldsymbol{H}\right]=I$. Correspondingly, the noise vector $\boldsymbol{n}$ is a multivariate complex Gaussian noise vector distributed with zero mean and a covariance matrix $E\left[\boldsymbol{n} \boldsymbol{n}^{H}\right]=2 \sigma^{2} \boldsymbol{I}_{N}$. For the DS-CDMA, $\sigma^{2}=1 /\left(2 \gamma_{b}\right)$ with $\gamma_{b}$ representing the average SNR per bit. For the SDMA, we have $\sigma^{2}=1 /\left(2 N \gamma_{b}\right)$, where $\gamma_{b}$ denotes the average SNR per bit per receive antenna.

In this paper, we explore mainly the BER performance of the DS-CDMA and SDMA systems using RMD/MS-MMSE MUD, when these systems are heavily loaded. Specifically, a system is referred to as a full-load system when $K=N$ and as an overload system if $K>N$. Otherwise, it is referred to as an under-load system if $K<N$.

When MMSE-MUD is employed, the decision variables for the $K$ users or, specifically, the decision variable for user $k, k=1, \ldots, K$, can be expressed as [10]

$$
\boldsymbol{z}=\Re\left\{\boldsymbol{W}^{H} \boldsymbol{y}\right\}, \quad z_{k}=\Re\left\{\boldsymbol{w}_{k}^{H} \boldsymbol{y}\right\},
$$

respectively, where $z=\left[z_{1}, z_{2}, \cdots, z_{K}\right]^{T}$, the $(N \times K)$ weight matrix $\boldsymbol{W}=\boldsymbol{R}_{y}^{-1} \boldsymbol{H}$ with $\boldsymbol{R}_{y}=\boldsymbol{H} \boldsymbol{H}^{H}+2 \sigma^{2} \boldsymbol{I}_{N}=$ $\sum_{k=1}^{K} \boldsymbol{h}_{k} \boldsymbol{h}_{k}^{H}+2 \sigma^{2} \boldsymbol{I}_{N}$ being the autocorrelation matrix of $\boldsymbol{y}$, while $\boldsymbol{w}_{k}=\left(1+\boldsymbol{h}_{k}^{H} \boldsymbol{R}_{k}^{-1} \boldsymbol{h}_{k}\right)^{-1} \boldsymbol{R}_{k}^{-1} \boldsymbol{h}_{k}$ with $\boldsymbol{R}_{k}=$ $\left(\sum_{l \neq k}^{K} \boldsymbol{h}_{l} \boldsymbol{h}_{l}^{H}+2 \sigma^{2} \boldsymbol{I}_{N}\right)$ being the autocorrelation matrix of the interference plus noise. 
According to [17], the decision variable $z_{k}$ can be approximated as a Gaussian random variable with its PDF given by

$$
f\left(z_{k} \mid b_{k}\right)=\frac{1}{\sqrt{2 \pi} \sigma_{k}} \exp \left[-\frac{\left(z_{k}-m_{k}\right)^{2}}{2 \sigma_{k}^{2}}\right], k=1,2, \ldots, K
$$

where the mean and variance are [10]

$$
m_{k}=\frac{\bar{\gamma}_{k}}{1+\bar{\gamma}_{k}} b_{k}, \quad \sigma_{k}^{2}=\frac{\bar{\gamma}_{k}}{2\left(1+\bar{\gamma}_{k}\right)^{2}}
$$

respectively. Furthermore, in (22), $\bar{\gamma}_{k}=\boldsymbol{h}_{k}^{H} \boldsymbol{R}_{k}^{-1} \boldsymbol{h}_{k}$ denotes the instantaneous signal-to-interference-plus-noise ratio (SINR) for detecting user $k$.

It can be shown [18] that, for both the DS-CDMA using random sequences and SDMA, any two decision variables $z_{i}$ and $z_{j}$, where $i \neq j$, become asymptotically independent, i.e., satisfying $f\left(z_{i}, z_{j} \mid b_{i}, b_{j}\right)=f\left(z_{i} \mid b_{i}\right) f\left(z_{j} \mid b_{j}\right)$, as $N \rightarrow \infty$. This is because, for any $i \neq j, \boldsymbol{h}_{i}^{H} \boldsymbol{h}_{j}=0$ with probability one, i.e., the signature sequences of users $i$ and $j$ become orthogonal with probability one, as $N \rightarrow \infty$. In fact, since for any linear detectors, such as correlation, zero-forcing, MMSE, etc. detectors [3], [4], the decision variables $z_{i}$ and $z_{j}$ are related via the terms $\boldsymbol{h}_{i}^{H} \boldsymbol{h}_{j}$ or $\boldsymbol{h}_{j}^{H} \boldsymbol{h}_{i}$, hence, the decision variables $z_{i}$ and $z_{j}$ become asymptotically independent, as $N \rightarrow \infty$. The only difference is that the decision variables in some detectors become independent faster than that in some other detectors.

\section{Receiver Multiuser Diversity Aided Multi-Stage MMSE Detection}

In this section, we first consider the characteristics of reliability measurement (RM), motivating to design lowcomplexity near-optimum RM schemes. Then, the RMD/MSMMSE MUD is addressed.

\section{A. Reliability Measurement in MMSE Detection}

Given the decision variable vector $z$, such as that in (20), the reliability of detecting $b_{k}$ can be optimally measured by the magnitude of the posteriori LLR [15]

$$
\begin{aligned}
L_{k} & =\left|\mathcal{L}_{k}\right|=\left|\ln \left[\frac{f\left(b_{k}=+1 \mid \boldsymbol{z}\right)}{f\left(b_{k}=-1 \mid \boldsymbol{z}\right)}\right]\right|=\left|\ln \left[\frac{f\left(\boldsymbol{z}, b_{k}=+1\right)}{f\left(\boldsymbol{z}, b_{k}=-1\right)}\right]\right| \\
& =\left|\ln \left[\frac{\sum_{\boldsymbol{b}_{k}^{+} \in\{+1,-1\}^{K}} f\left(\boldsymbol{z}, \boldsymbol{b}_{k}^{+}\right)}{\sum_{\boldsymbol{b}_{k}^{-} \in\{+1,-1\}^{K}} f\left(\boldsymbol{z}, \boldsymbol{b}_{k}^{-}\right)}\right]\right|, k=1, \ldots, K \quad(23)
\end{aligned}
$$

where $\boldsymbol{b}_{k}^{+}$and $\boldsymbol{b}_{k}^{-}$are $K$-length binary vectors with their $k$ th entries being +1 and -1 , respectively. Note that, $b_{k}$ is optimally detected by the sign of $\mathcal{L}_{k}$ in the sense of MAP.

From (23) and the principles of MAP [15], we can have the following two observations. First, if $z$ can be divided into two independent vectors $z_{a}$ related to $K_{1}$ users and $z_{b}$ related to the rest $\left(K-K_{1}\right)$ users, then, assuming that $b_{k}$ is associated with $z_{a}$, we can readily show that $L_{k}$ can be evaluated as

$$
L_{k}=\left|\ln \left[\frac{\sum_{\boldsymbol{b}_{k}^{+} \in\{+1,-1\}^{K_{1}}} f\left(\boldsymbol{z}_{a}, \boldsymbol{b}_{k}^{+}\right)}{\sum_{\boldsymbol{b}_{k}^{-} \in\{+1,-1\}^{K_{1}}} f\left(\boldsymbol{z}_{a}, \boldsymbol{b}_{k}^{-}\right)}\right]\right|
$$

Eq.(24) explains that the detection of $b_{k}$ or the corresponding $\mathrm{RM}$ is independent of the bits related to $z_{b}$. Hence, the reliabilities of the users with independent sets of decision variables can be measured independently. Furthermore, in the extreme case where $z_{k}$ is independent of all the other decision variables, we then have $L_{k}=\left|\mathcal{L}_{k}\right|=\left|\ln \left[\frac{f\left(z_{k}, b_{k}=+1\right)}{f\left(z_{k}, b_{k}=-1\right)}\right]\right|$, implying that the optimum detection of user $k$ is independent of all the other users.

Second, if the $m$ th, $m \neq k$, user has been detected correctly, (23) becomes

$$
\begin{aligned}
& L_{k}=\left|\mathcal{L}_{k}\right|=\left|\ln \left[\frac{\sum_{\boldsymbol{b}_{k, m}^{+} \in\{+1,-1\}^{K}} f\left(\boldsymbol{z}, \boldsymbol{b}_{k, m}^{+}\right)}{\sum_{\boldsymbol{b}_{k, m}^{-} \in\{+1,-1\}^{K}} f\left(\boldsymbol{z}, \boldsymbol{b}_{k, m}^{-}\right)}\right]\right|, \\
& k=1,2, \ldots, K, ; k \neq m
\end{aligned}
$$

where $\boldsymbol{b}_{k, m}^{+}$and $\boldsymbol{b}_{k, m}^{-}$are $K$-length binary vectors with their $k$ th entries being +1 and -1 , while with their $m$ th entries replaced by $\hat{b}_{m}$ of the estimate to $b_{m}$. Equation (25) implies that, once a user has been detected correctly, then cancellation of the detected user is optimum to the other not yet detected users.

From Eqs. (24) and (25), we can gain the following insights. First, when the decision variables of $K$ users become more important, the separate detection of the $K$ users becomes more optimum towards the optimum MAP detection. Second, in order to benefit from the low-complexity SIC-MUDs but experience the least effect of error propagation, the reliabilities corresponding to different users should be highly diverse, showing that some user signals are much more reliable than some other user signals. In this case, a more reliable user signal can be detected before a less reliable user signal and its interference on the less reliable user signal can be canceled with high confidence.

As we state in Section III, in the MMSE-MUD, any two decision variables become asymptotically independent, as $N \rightarrow \infty$. Furthermore, according to [17], the decision variables provided by the MMSE-MUD converge to the Gaussian random variables, as $N \rightarrow \infty$. Hence, for a given value of $N$, when we approximate the decision variables $z_{1}, z_{2}, \ldots, z_{K}$, as shown in (20), as the iid random variables obeying the Gaussian distribution as shown in (21), the reliability of (23) can be simplified to

$$
L_{k} \approx\left|\ln \left[\frac{f\left(z_{k}, b_{k}=+1\right)}{f\left(z_{k}, b_{k}=-1\right)}\right]\right|=\left|\ln \left[\frac{f\left(z_{k} \mid b_{k}=+1\right)}{f\left(z_{k} \mid b_{k}=-1\right)}\right]\right|
$$

where the second equality is owing to the assumption of $P\left(b_{k}=+1\right)=P\left(b_{k}=-1\right)$. Furthermore, when applying the PDF of (21) into (26), the reliability for detecting user $k$ in MAP sense can be expressed as ${ }^{1}[10]$

$$
\begin{aligned}
L_{k} & =4\left(1+\bar{\gamma}_{k}\right)\left|z_{k}\right| \triangleq\left(1+\bar{\gamma}_{k}\right)\left|z_{k}\right| \\
& =\left(1-\boldsymbol{h}_{k}^{H} \boldsymbol{R}_{y}^{-1} \boldsymbol{h}_{k}\right)^{-1}\left|z_{k}\right|
\end{aligned}
$$

This equation shows that, after the MMSE-MUD, the reliabilities can be directly evaluated by invoking the auto-correlation matrix $\boldsymbol{R}_{y}$, which in practice can be readily estimated from

\footnotetext{
${ }^{1}$ If the source symbols do not satisfy $P\left(b_{k}=+1\right)=P\left(b_{k}=-1\right)$, the reliability in MAP sense can be derived by the first equation in (26), resulting in $L_{k}=\left|4\left(1+\bar{\gamma}_{k}\right) z_{k}+L_{0}\right|, k=1,2, \ldots, K$, where $L_{0}=$ $\ln \left[P\left(b_{k}=+1\right) / P\left(b_{k}=-1\right)\right]$ denotes the a-priori information of $b_{k}$. In this case, (27) is the reliability measured in the sense of ML.
} 


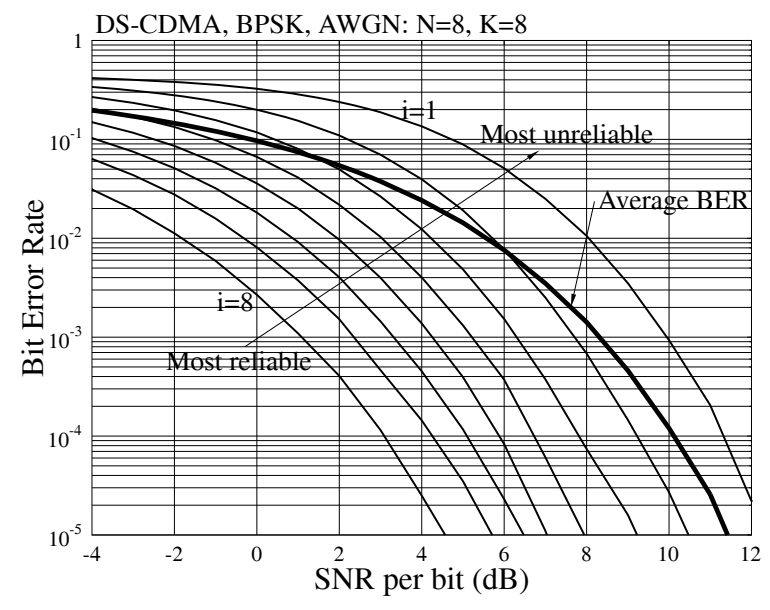

Fig. 2. BER versus SNR per bit $\left(\gamma_{b}\right)$ for the bits at different reliability levels detected by the MMSE-MUD in a DS-CDMA system using BPSK modulation, when communicating over Gaussian channels.

the observations obtained at receiver. Furthermore, when the $K$ reliabilities are required to be computed simultaneously, we can express $\boldsymbol{Q}=\boldsymbol{I}_{K}-\boldsymbol{H}^{H} \boldsymbol{R}_{y}^{-1} \boldsymbol{H}$ and re-write (27) as $L_{k}=\boldsymbol{Q}^{-1}(k, k)\left|z_{k}\right|$, where $\boldsymbol{Q}(k, k)$ denotes the $(k, k)$ th entry of $Q$.

Note that, since the decision variables $z_{1}, z_{2}, \ldots, z_{K}$ become more independent, as the value of $N$, which determines the size of a DS-CDMA or SDMA system, increases. Hence, as the system size increases, the RM based on (27) converges to the optimum RM based on (23). This property is also illustrated by our simulation results in Section $\mathrm{V}$ and those in [11]. Furthermore, the LLR-assisted RM based on (27) takes into account of both the instantaneous SINR $\bar{\gamma}_{k}$ and the magnitude of the decision variable $z_{k}$ containing real-time Gaussian noise. This RM scheme is valid for operation in any communications scenarios (such as MUD, inter-symbol interference (ISI) suppression, V-BLAST, error-correction decoding, etc.) and over any communications channels (fading or non-fading).

Based on the RM of (27), Fig. 2 shows the ordered BER of the DS-CDMA systems communicating over Gaussian channels. The ordered BER for the SDMA systems can be found in [10]. Explicitly, after the MMSE-MUD, the BER of the users at different reliability levels is highly diverse. Hence, significant RMD is revealed for both the DS-CDMA and SDMA systems employing the MMSE-MUD, when communicating over either Gaussian or Rayleigh fading channels. Furthermore, when comparing Fig. 2 with Fig. 1 derived based on $K$ independent decision variables, we can observe that the ordered BER performance shown in both figures is very similar, except a slight shift on the SNR axis. This observation explains that, after the MMSE-MUD, the decision variables become highly independent. The slight shift of BER performance is the result of the correlation not removed by the MMSE-MUD.

\section{B. RMD/MS-MMSE MUD Algorithm}

From the previous analysis, we can know that the MMSEMUD aided by the LLR-based RM employs the two properties required for implementing the high-efficiency SIC-MUD, as discussed in Section IV-A. Therefore, the RMD/MS-MMSE MUD is proposed, which exploits the embedded RMD to determine a detection order and is operated in the principles similar to that of the V-BLAST systems [7]-[9]. The RMD/MS-MMSE MUD is divided into $K$ detection stages (although can be modified in different ways [10]), each stage detects one user that is assumed to be the most reliable of the users having not been detected yet, whose reliabilities are measured according to (27).

Let $\boldsymbol{R}_{y}^{(0)}=\boldsymbol{R}_{y}$ express the autocorrelation matrix of $\boldsymbol{y}^{(0)}=\boldsymbol{y}$, and $\boldsymbol{R}_{y}^{(s)}$ express the autocorrelation matrix of $\boldsymbol{y}^{(s)}$ of the output generated by the $s$ th detection stage under the assumption that the previous $(s-1)$ stages of detection are correct. Let the signature of the user detected at the sth stage be denoted by $\boldsymbol{h}^{(s)}$. Furthermore, let after the $s$ th detection stage the signatures of the users having not been detected be collected in $\tilde{\boldsymbol{H}}^{(s)}$, where $\tilde{\boldsymbol{H}}^{(0)}=\boldsymbol{H}$. Then, the RMD/MSMMSE MUD can be described as follows:

Initialization: $\boldsymbol{y}^{(0)}=\boldsymbol{y}, \boldsymbol{R}_{y}^{(0)}=\boldsymbol{R}_{y}, \tilde{\boldsymbol{H}}^{(0)}=$ $\boldsymbol{H}, \boldsymbol{W}^{(0)}=\boldsymbol{R}_{y}^{-1} \boldsymbol{H}, \boldsymbol{Q}^{(0)}=\boldsymbol{I}_{K}-\left(\tilde{\boldsymbol{H}}^{(0)}\right)^{H} \boldsymbol{W}^{(0)} ;$

Detection: for $s=1,2, \ldots, K$,

1) Forming decision variables: $\boldsymbol{z}^{(s)}=\Re\left\{\left(\boldsymbol{W}^{(s-1)}\right)^{H} \boldsymbol{y}^{(s-1)}\right\}$

2) Determining the most reliable user: For the users $k_{1}^{\prime}, k_{2}^{\prime}, \ldots, k_{K-s+1}^{\prime}$ that have not been detected, compute their reliabilities according to (27), and find the most reliable user of $k^{(s)}=\arg \max _{k_{i}^{\prime}}\left\{L_{k_{1}^{\prime}}, L_{k_{2}^{\prime}}, \ldots, L_{k_{K-s+1}^{\prime}}\right\}$;

3) Detection of the most reliable: $\hat{b}^{(s)}=$ $\operatorname{sgn}\left(z_{k^{(s)}}^{(s)}\right)$, where $z_{k^{(s)}}^{(s)}$ is the $k^{(s)}$ th entry of $\boldsymbol{z}^{(s)}$

4) Interference cancellation: $\boldsymbol{y}^{(s)}=\boldsymbol{y}^{(s-1)}-$ $\boldsymbol{h}^{(s)} \hat{b}^{(s)}$

5) Update:

$$
\begin{aligned}
& \boldsymbol{W}^{(s)}=\left[\boldsymbol{W}^{(s-1)}+\frac{\boldsymbol{w}^{(s-1)}\left(\boldsymbol{h}^{(s)}\right)^{H} \boldsymbol{W}^{(s-1)}}{\boldsymbol{Q}^{(s-1)}\left(k^{(s)}, k^{(s)}\right)}\right] \boldsymbol{P}^{(s)}, \\
& \tilde{\boldsymbol{H}}^{(s)}=\tilde{\boldsymbol{H}}^{(s-1)} \boldsymbol{P}^{(s)}, \\
& \boldsymbol{Q}^{(s)}=\boldsymbol{I}-\left(\tilde{\boldsymbol{H}}^{(s)}\right)^{H} \boldsymbol{W}^{(s)} .
\end{aligned}
$$

In (28), $\boldsymbol{Q}^{(s-1)}\left(k^{(s)}, k^{(s)}\right)$ is the $\left(k^{(s)}, k^{(s)}\right)$ th entry of $\boldsymbol{Q}^{(s-1)}$, while $\boldsymbol{w}^{(s-1)}$ is the $k^{(s)}$ th column of $\boldsymbol{W}^{(s-1)}$. Furthermore, $\tilde{\boldsymbol{H}}^{(s)}$ is obtained from $\tilde{\boldsymbol{H}}^{(s-1)}$ by deleting the column of $\boldsymbol{h}^{(s)}$ corresponding to the user detected at the sth detection stage, and $\boldsymbol{P}^{(s)}$ is a permutation matrix obtained from $\boldsymbol{I}_{K}$ after removing the $s$ columns corresponding to the users having been detected.

The RMD/MS-MMSE MUD can be efficiently implemented using the existing algorithms proposed in the context of the V-BLAST detection [12]-[14]. It can be shown that the complexity of the RMD/MS-MMSE MUD is on the order of $\mathcal{O}\left(c_{1} K N+c_{2} N^{2}\right)$ per user, where $c_{1}$ and $c_{2}$ are certain constants. Therefore, for a given $N$, the complexity of the RMD/MS-MMSE MUD increases linearly with $K$. The detection delay of the RMD/MS-MMSE MUD is also linearly 


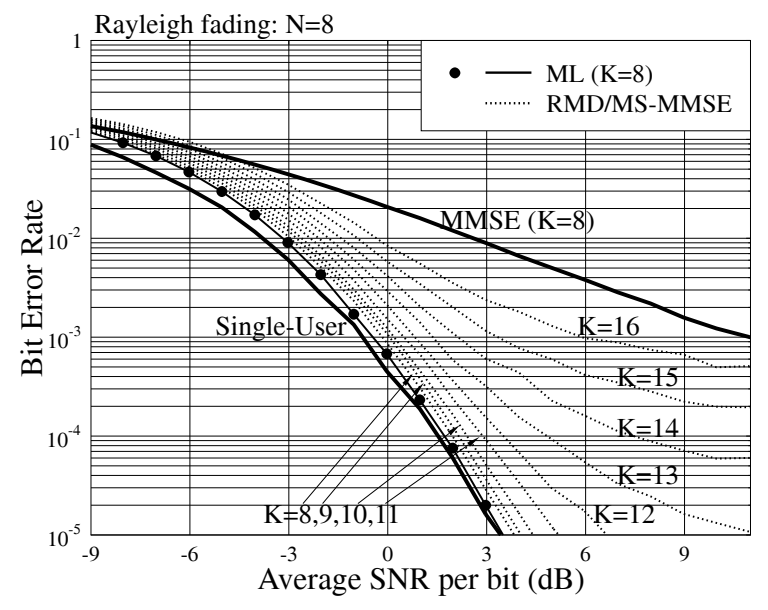

Fig. 3. BER versus average SNR per bit performance of the SDMA systems communicating over Rayleigh fading channels.

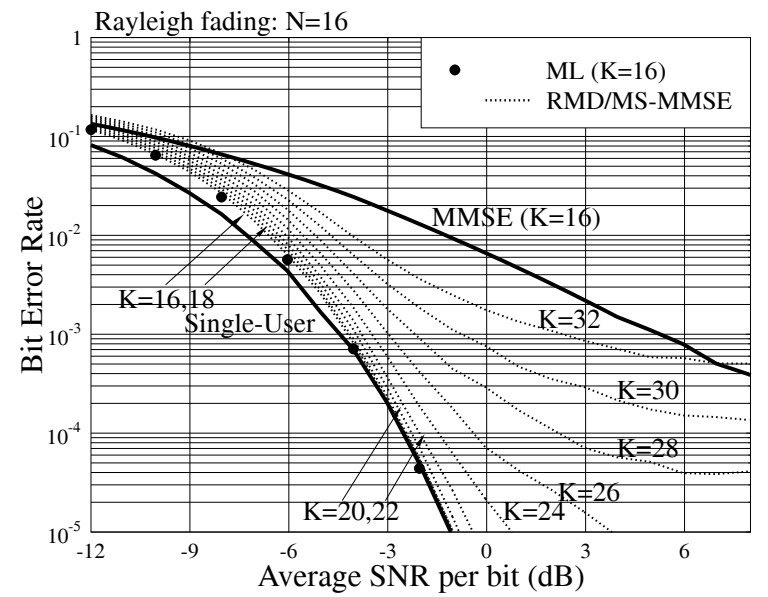

Fig. 4. BER versus average SNR per bit performance of the SDMA systems communicating over Rayleigh fading channels.

depended on $K$. However, the RMD/MS-MMSE MUD is a symbol-by-symbol detector. Hence, its maximum detection delay is $K$ symbols.

\section{Performance Results}

In this section, simulation results are provided to illustrate the achievable BER performance of the RMD/MS-MMSE MUD, when it is employed in DS-CDMA or SDMA systems. The BER performance results for the SDMA systems are shown in Figs. 3 and 4, while that for the DS-CDMA systems are shown in Figs. 5 and 6. For the sake of comparison, a range of benchmark BER results were also provided. Specifically, in all these figures the single-user BER bounds corresponding to $K=1$ and the BER of the MMSE-MUD for the fullload $(K=N)$ DS-CDMA or SDMA systems were depicted. Additionally, in Figs. 3 and 4, the BER of the ML-MUD for the full-load systems was also illustrated. From the BER performance results shown in Figs. 3 - 6, we can have the following observations.

First, the RMD/MS-MMSE MUD is suitable for diverse communications scenarios. It is highly efficient for both the

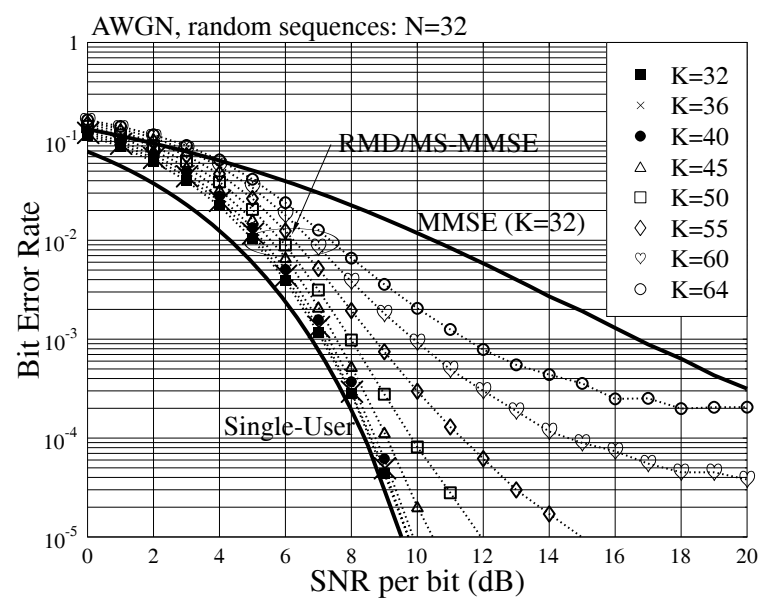

Fig. 5. BER versus average SNR per bit performance of the DS-CDMA systems communicating over AWGN channels.

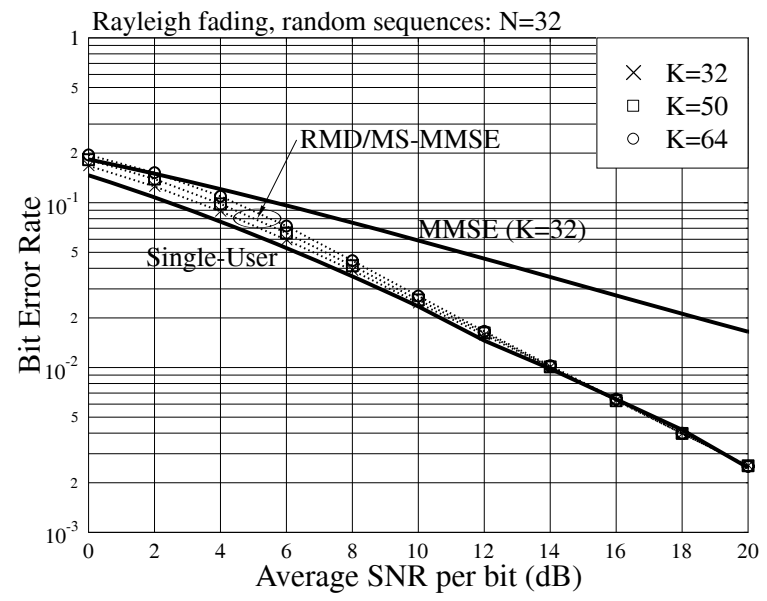

Fig. 6. BER versus average SNR per bit performance of the DS-CDMA systems communicating over flat Rayleigh fading channels.

DS-CDMA and SDMA systems. It is suitable for both Gaussian and fading channels. Furthermore, it can be applied for detection in the multiuser systems that are under-load, fullload or overload.

Second, when the DS-CDMA or SDMA systems are fullload, i.e., when $K=N$, the RMD/MS-MMSE MUD is generally capable of achieving the BER performance near that of the ML-MUD. As shown in Fig. 3, the BER performance of the RMD/MS-MMSE MUD for the case of $K=N=8$ is very close to that achieved by the ML-MUD. In Fig. 4, the BER of the RMD/MS-MMSE MUD for $K=N=16$ is nearly the same as that of the ML-MUD. Similarly, in Figs. 5 and 6, the BER achieved by the RMD/MS-MMSE MUD for the cases $K=N$ is either the same as or very close to the single-user BER bound. In fact, for the full-load cases, we can deduce from the shapes of the BER curves in Figs. 5 and 6 and from their locations relative to the single-user bounds that the RMD/MS-MMSE MUD has achieved nearly the same BER performance as the ML-MUD.

Third, for all the scenarios considered, at a BER of $10^{-2}$, which is often the BER interested in practical systems before error-correction decoding, the BER performance of 
the RMD/MS-MMSE MUD for the DS-CDMA or SDMA systems supporting $K=2 \mathrm{~N}$ users is much better than that of the MMSE-MUD for the DS-CDMA or SDMA systems supporting only $K=N$ users. Furthermore, at a BER of $10^{-2}$, as shown in Figs. $3-6$, the number of users supported can be doubled from $K=N$ to $K=2 N$ by increasing the SNR per bit less than $3 \mathrm{~dB}$. Therefore, with the RMD/MS-MMSE MUD, the throughput of DS-CDMA or SDMA systems increases at least linearly with the SNR per bit invested.

\section{CONCLUSIONS}

We have shown that, for a sufficiently large multiuser system, the MMSE-MUD is capable of providing nearindependent detection of signals and generating significant RMD, when measured in MAP sense. Consequently, the RMD/MS-MMSE MUD designed by making use of these properties is highly efficient for operation in both full-load and overload systems. Aided by the RMD/MS-MMSE MUD, a full-load DS-CDMA or SDMA system of moderate size is generally capable of achieving the BER performance similar to that of the ML-MUD. Within the BER range of interest, an overload DS-CDMA or SDMA system using the RMD/MSMMSE MUD to support $K=2 N$ users can still significantly outperform a corresponding DS-CDMA or SDMA system using the MMSE-MUD to support $K=N$ users. Furthermore, the proposed RMD/MS-MMSE MUD's complexity is at the level similar to a conventional MMSE-SIC scheme or a VBLAST detection scheme.

Note that the RMD/MS-MMSE MUD may be modified in different ways, in order to obtain a desirable balance among the achievable BER performance, implementation complexity and detection delay. For example, at one detection stage several users with their reliabilities higher than a pre-set threshold may be detected simultaneously, in order to reduce the detection delay. Another example is that, in an overload system, if more complexity is affordable, the last several users may be detected using MAP or ML MUD, in order to lower the error-floors as seen in Figs. 3 - 6. Note additionally that, following this paper, the RMD/MS-MMSE MUD has been investigated in the context of non-binary modulation schemes, the details of which can be found in [19].

\section{APPENDIX \\ Derivation of The Ordered Bit ERror Rate}

Let us assume $b_{k}=-1$ is transmitted by user $k$. Then, the BER of the $i$ th most unreliable user can be expressed as

$$
\begin{aligned}
P_{E}(i)= & K\left(\begin{array}{c}
K-1 \\
i-1
\end{array}\right) P\left(y_{k}>0 \mid L_{(i)}=L_{k}, b_{k}=-1\right) \\
& \times\left[P\left(L_{m} \leq L_{k}\right)\right]^{i-1}\left[P\left(L_{n} \geq L_{k}\right)\right]^{K-i}
\end{aligned}
$$

where $P\left(y_{k}>0 \mid L_{(i)}=L_{k}, b_{k}=-1\right)$ denotes the probability of $y_{k}>0$, i.e., error probability, given that the $k$ th user is the $i$ th most unreliable, while the terms of $\left[P\left(L_{m} \leq L_{k}\right)\right]^{i-1}$ and $\left[P\left(L_{n} \geq L_{k}\right)\right]^{K-i}$ mean that there are $(i-1)$ users are less reliable and $(K-i)$ users are more reliable than user $k$. Since $y_{k}>0$ and $q_{k}>0$ are equivalent events and, furthermore, in this case $L_{k}=\left|q_{k}\right|=q_{k}$, from (29) we obtain

$$
\begin{aligned}
P_{E}(i)= & K\left(\begin{array}{c}
K-1 \\
i-1
\end{array}\right) P\left(q_{k}>0 \mid L_{(i)}=q_{k}, b_{k}=-1\right) \\
& \times\left[P\left(L_{m} \leq q_{k}\right)\right]^{i-1}\left[P\left(L_{n} \geq q_{k}\right)\right]^{K-i} \\
= & K\left(\begin{array}{c}
K-1 \\
i-1
\end{array}\right) \int_{0}^{\infty} f_{q_{k}}\left(y \mid b_{k}=-1\right) \\
& \times\left[F_{L_{m}}(y)\right]^{i-1}\left[1-F_{L_{n}}(y)\right]^{K-i} d y
\end{aligned}
$$

where $L_{m}, L_{n} \in\left\{L_{1}, \ldots, L_{k-1}, L_{k+1} \ldots, L_{K}\right\}$, while $f_{q_{k}}\left(y \mid b_{k}=-1\right)$ is given in (4) for Gaussian channels and in (9) for Rayleigh fading channels. Hence, when substituting (4) with $b_{k}=-1$ and $\gamma_{k}=\gamma_{b}$ as well as (12) into (30), we obtain for Gaussian channels

$$
\begin{aligned}
P_{E}(i) & =K\left(\begin{array}{c}
K-1 \\
i-1
\end{array}\right) \frac{1}{\sqrt{\pi \gamma_{b}}} \int_{0}^{\infty} \exp \left(-\frac{\left(y+\gamma_{b}\right)^{2}}{\gamma_{b}}\right) \\
& \times\left[1-Q\left(\sqrt{\frac{2}{\gamma_{b}}} y+\sqrt{2 \gamma_{b}}\right)-Q\left(\sqrt{\frac{2}{\gamma_{b}}} y-\sqrt{2 \gamma_{b}}\right)\right]^{i-1} \\
& \times\left[Q\left(\sqrt{\frac{2}{\gamma_{b}}} y+\sqrt{2 \gamma_{b}}\right)+Q\left(\sqrt{\frac{2}{\gamma_{b}}} y-\sqrt{2 \gamma_{b}}\right)\right]^{K-i} d y
\end{aligned}
$$

Let us in the above equation use the variable transform $\left(y+\gamma_{b}\right) / \sqrt{\gamma_{b}}=t / \sqrt{2}$. Then, the BER of the bits at the $i$ th reliability level over Gaussian channels can be simplified to (16).

In the context of the Rayleigh fading channels, substituting (9) with $b_{k}=-1$ and (14) into (30) yields (32). See top of next page. Upon invoking the variable transform of $2\left[1+\sqrt{\left(1+\gamma_{b}\right) / \gamma_{b}}\right] y=t$, one can obtain (33). See top of next page.

Finally, after using the binomial theorem to expand the brackets invoked and completing the integration, the BER of the bits at the $i$ th reliability level for communications over Rayleigh fading channels can be formulated as (17).

\section{REFERENCES}

[1] S. Verdu, "Minimum probability of error for asynchronous multiple access communication systems," in Proc. IEEE Military Commun. Conf., Oct. 1983 , pp. 213-219.

[2] — - "Minimum probability of error for asynchronous Gaussian multiple-access channels," IEEE Trans. Inf. Theory, vol. 32, no. 1, pp. 85-96, Jan. 1986.

[3] _ Multiuser Detection. Cambridge University Press, 1998.

[4] L.-L. Yang, Multicarrier Communications. Chichester, UK: John Wiley, 2009.

[5] F. Hasegawa, et al., "Speed and accuracy comparison of techniques for multiuser detection in synchronous CDMA," IEEE Trans. Commun., vol. 52, no. 4, pp. 540-545, Apr. 2004.

[6] A. Duel-Hallen, "A family of multiuser decision-feedback detectors for asynchronous code-division multiple-access channels," IEEE Trans. Commun., vol. 43, no. 2/3/4, pp. 421 -434, Feb./Mar./Apr. 1995.

[7] P. Wolniansky, G. Foschini, G. Golden, and R. Valenzuela, "V-BLAST: an architecture for realizing very high data rates over the rich-scattering wireless channel," in Proc. IEEE Inter. Symposium Sig., Sys., Elec. (ISSSE), 1998, pp. 295-300.

[8] G. Foschini, G. Golden, R. Valenzuela, and P. Wolniansky, "Simplified processing for high spectral efficiency wireless communication employing multi-element arrays," IEEE J. Sel. Areas Commun., vol. 17, no. 11, pp. 1841-1851, Nov. 1999.

[9] G. Golden, C. Foschini, R. Valenzuela, and P. Wolniansky, "Detection algorithm and initial laboratory results using V-BLAST space-time communication architecture," IEE Electron. Lett., vol. 35, no. 1, pp. 14-16, Jan. 1999. 


$$
\begin{aligned}
P_{E}(i) & =K\left(\begin{array}{c}
K-1 \\
i-1
\end{array}\right) \sqrt{\frac{1}{\gamma_{b}\left(1+\gamma_{b}\right)}} \int_{0}^{\infty} \exp \left(-2\left[1+\sqrt{\frac{1+\gamma_{b}}{\gamma_{b}}}\right] y\right) \\
\times & {\left[1-\frac{1}{2}\left(1-\sqrt{\frac{\gamma_{b}}{1+\gamma_{b}}}\right) \exp \left(-2\left[1+\sqrt{\frac{1+\gamma_{b}}{\gamma_{b}}}\right] y\right)-\frac{1}{2}\left(1+\sqrt{\frac{\gamma_{b}}{1+\gamma_{b}}}\right) \exp \left(2\left[1-\sqrt{\frac{1+\gamma_{b}}{\gamma_{b}}}\right] y\right)\right]^{i-1} } \\
\times & {\left[\frac{1}{2}\left(1-\sqrt{\frac{\gamma_{b}}{1+\gamma_{b}}}\right) \exp \left(-2\left[1+\sqrt{\frac{1+\gamma_{b}}{\gamma_{b}}}\right] y\right)+\frac{1}{2}\left(1+\sqrt{\frac{\gamma_{b}}{1+\gamma_{b}}}\right) \exp \left(2\left[1-\sqrt{\frac{1+\gamma_{b}}{\gamma_{b}}}\right] y\right)\right]^{K-i} d y }
\end{aligned}
$$

$$
\begin{aligned}
& P_{E}(i)=\frac{K\left(\begin{array}{c}
K-1 \\
i-1
\end{array}\right)}{2\left(\sqrt{\gamma_{b}\left(1+\gamma_{b}\right)}+\gamma_{b}+1\right)} \int_{0}^{\infty} \exp (-t) \times\left[1-\frac{1}{2}\left(1-\sqrt{\frac{\gamma_{b}}{1+\gamma_{b}}}\right) \exp (-t)\right. \\
&-\left.\frac{1}{2}\left(1+\sqrt{\frac{\gamma_{b}}{1+\gamma_{b}}}\right) \exp \left(\left[2 \sqrt{\gamma_{b}\left(1+\gamma_{b}\right)}-2 \gamma_{b}-1\right] t\right)\right]^{i-1} \\
& \times {\left[\frac{1}{2}\left(1-\sqrt{\frac{\gamma_{b}}{1+\gamma_{b}}}\right) \exp (-t)+\frac{1}{2}\left(1+\sqrt{\frac{\gamma_{b}}{1+\gamma_{b}}}\right) \times \exp \left(\left[2 \sqrt{\gamma_{b}\left(1+\gamma_{b}\right)}-2 \gamma_{b}-1\right] t\right)\right]^{K-i} d t, } \\
& i=1,2, \ldots, K
\end{aligned}
$$

[10] L.-L. Yang, "Using multi-stage MMSE detection to approach optimum error performance in multiantenna MIMO systems," in Proc. IEEE 70th Veh. Technol. Conf. (VTC-Fall), pp. 20-23 Sep. 2009.

[11] — "Receiver multiuser diversity aided multi-stage MMSE multiuser detection: a low-complexity detector fast-converging to the optimum," in Proc. IEEE 71th Veh. Technol. Conf. (VTC-Spring), May 2010.

[12] J. Benesty, Y. Huang, and J. Chen, "A fast recursive algorithm for optimum sequential signal detection in a BLAST system," IEEE Trans. Signal Process., vol. 51, no. 7, pp. 1722-1730, July 2003.

[13] K. Kusume, M. Joham, W. Utschick, and G. Bauch, "Cholesky factorization with symmetric permutation applied to detecting and precoding spatially multiplexed data streams," IEEE Trans. Signal Process., vol. 55, no. 6, pp. 3089-3103, June 2007.

[14] T. Liu and Y.-L. Liu, "Modified fast recursive algorithm for efficient MMSE-SIC detection of the V-BLAST system," IEEE Trans. Wireless Commun., vol. 7, no. 10, pp. 3713-3717, Oct. 2008.
[15] J. Hagenauer, E. Offer, and L. Papke, "Iterative decoding of binary block and convolutional codes," IEEE Trans. Inf. Theory, vol. 42, no. 2, pp. 429-445, Mar. 1996.

[16] I. Gradshteyn and I. Ryzhik, Table of Integrals, Series, and Products. New York: Academic Press, Inc, 1980.

[17] H. V. Poor and S. Verdu, "Probability of error in MMSE multiuser detection," IEEE Trans. Inf. Theory, vol. 43, no. 3, pp. 858-871, May 1997.

[18] A. M. Tulino and S. Verdu, Random Matrix Theory and Wireless Communications. now Publishers Inc., 2004.

[19] L.-L. Yang, "Receiver multiuser diversity aided multi-stage MMSE multiuser detection for DS-CDMA and SDMA systems employing I-Q modulation," in Proc. IEEE 72th Veh. Technol. Conf. (VTC-Fall), Sep. 2010. 